\title{
In vivo fluoroscopic kinematography of dynamic radio-ulnar incongruence in dogs
}

\author{
Thomas Rohwedder ${ }^{1, *}$, Martin Fischer ${ }^{2}$ and Peter Böttcher ${ }^{1}$ \\ ${ }^{1}$ Department of Small Animal Medicine, University of Leipzig, An den Tierkliniken 23, 04103 Leipzig, Germany \\ ${ }^{2}$ Institute of Systematic Zoology and Evolutionary Biology with Phyletic Museum, Friedrich-Schiller-University, \\ Jena, Germany
}

\begin{abstract}
Aim of the study was to investigate dynamic radio-ulnar incongruence (dRUI) in the canine elbow joint comparing orthopedic healthy and dysplastic dogs in a prospective in vivo study design. In 6 orthopedic sound elbow joints (5 dogs, median age 17 months \& mean body weight $27.9 \mathrm{~kg})$ and 7 elbow joints with medial coronoid disease $(6 \mathrm{dogs}$, median age 17.5 months \& mean body weight $27.6 \mathrm{~kg}$ ) $0.8 \mathrm{~mm} \varnothing$ tantalum beads were surgically implanted into radius, ulna and humerus for dynamic radiosteriometric analysis (RSA) using high-speed biplanar fluoroscopy with the dogs walking on a treadmill. dRUI, in the form of proximo-distal translation of the radius relative to the ulna, was measured for the first third of stance phase and compared between groups using unpaired t-testing. Healthy elbow joints exhibited a relative radio-ulnar translation of $0.7 \mathrm{~mm}(\mathrm{SD} 0.31 \mathrm{~mm})$, while dysplastic joints showed a translation of $0.5 \mathrm{~mm}(\mathrm{SD} 0.30 \mathrm{~mm})$. No significant difference between groups was detected $(\mathrm{p}=0.2092$, confidence interval $0.6-0.2)$. Based on these findings dRUI is present in every canine elbow joint, as part of the physiological kinematic pattern. However, dysplastic elbow joints do not show an increased radio-ulnar translation, and therfore dRUI cannot be considered causative for medial coronoid disease.
\end{abstract}

Keywords: Canine, Elbow dysplasia, Fluoroscopy, Gait analysis, Radio-ulnar incongruence.

\section{Introduction}

Developmental elbow disease is one of the most frequent causes of front limb lameness especially in young dogs, being bilateral in up to 35\% (Kirberger and Fourie, 1998; Morgan et al., 1999). Radio-ulnar incongruence (RUI) has been reported to occur concomitant to medial coronoid disease in up to $60 \%$ of clinical cases and has been shown to be related to the severity of medial compartment pathology (Eljack and Bottcher, 2015). Traditionally RUI has been defined as two forms of incongruence: (1) an abnormal shape of the trochlear notch of the ulna, leading to humero-ulnar incongruence (Wind, 1986; Wind and Packard, 1986) or (2) a step formation between the articular surfaces of the radius and ulna (short ulna or short radius), leading to radio-ulnar incongruence and secondary to this humero-ulnar conflict. Based on an ex vivo study we assume that under physiologic conditions joint forces are equally distributed between the radial head and the medial coronoid process (Mason et al., 2005). A static positive radio-ulnar incongruence (short radius) induces load shift from the radial head to the medial coronoid process (Krotscheck et al., 2014b; McConkey et al., 2016), with the consequence of mechanical overload of the medial joint compartment and degenerative changes such as fragmentation of the medial coronoid process (Samoy et al., 2006; Gemmill and Clements, 2007). According to Eljack and Bottcher (2015) we refer to RUI as "axial radio-ulnar incongruence", in contrast to local positive radio-ulnar incongruence at the tip of the medial coronoid process. Axial static RUI is thought to develop because of asynchronous growth between the radius and ulna (Samoy et al., 2006; Lau et al., 2015; Nemanic et al., 2016). However, in a significant number of dogs with medial coronoid disease RUI is not present (Gemmill et al., 2005; Kramer et al., 2006; Eljack and Bottcher, 2015). This might be due to transient RUI during growth, with incongruence having resolved by the time of clinical presentation, which is usually months later. Alternatively, RUI might occur dynamically when the joint is loaded during physical activity, resulting in false findings when the joint is evaluated for RUI in unloaded state, which is usually the case with dogs under anesthesia. There are two possible dynamic RUI scenarios: a radio-ulnar joint cup being congruent while unloaded becomes incongruent under load, and on the other hand, static RUI might become equalized when loaded. Both have been theorized by numerous investigators but remained unproven, until Guillou $e t$ al. (2011) confirmed in vivo dynamic axial radio-ulnar translation of up to $0.93 \mathrm{~mm}$ in sound canine elbow joints while the dogs were walking and trotting on a treadmill. Expecting a higher degree of dynamic RUI 
necessary to cause fissuring or fragmentation of the medial coronoid process in dysplastic dogs, we hypothesized that dynamic axial RUI will be siginificantly greater in dysplastic elbow joints when compared to normals. Therfore, it was the aim of the current study to compare the amount of dynamic RUI in orthopedic healthy and dysplastic canine elbow joints while the dogs were walking on a treadmill using three-dimensional fluoroscopic kinematography.

\section{Materials and Methods}

\section{Animals}

Only orthopedic healthy, adult, mid to large breed dogs $(20-35 \mathrm{~kg})$ were included in the control group. Dogs were defined to be undiseased if no history of lameness was present, complete orthopedic examination, biplanar radiographs and computed tomography (CT) of both elbow joints were inconspicuous of any pathology. Radiographs were assessed according to the International Elbow Working Group (IEWG) protocol (Ohlerth et al., 2016). By definition, the IEWG score had to be zero, without any evidence of subtrochlear sclerosis.

Similar to the control group only mid to large breed dogs $(20-35 \mathrm{~kg})$ with closed physis were considered for the dysplastic group. Dogs had to be lame because of elbow pain, either uni- or bilaterally. Orthopedic and radiologic examination had to confirm evidence of medial coronoid disease for further enrollment. As for the control dogs, radiographic assessment of elbow pathology was done according to the IEWG protocol. Any orthopedic abnormality other than medial coronoid disease (not including OCD) was defined to be an exclusion criteria as well as any abnormal finding during physical examination other than elbow pain and effusion. Exclusion and inclusion criteria had to be confirmed by CT and arthroscopy of the index joint(s). Radiographic and CT findings indicative for medial compartment disease on the contralateral elbow in case of unilateral lameness did not result in exclusion of the patient. The decision to include both or only one elbow joint in the study was based solely on clinical indication for arthroscopic evaluation and treatment, which was defined to be bilateral or unilateral elbow lameness, respectively.

All owners were informed about the purpose of the study and had to provide signed consent. The study was further approved by the local governmental ethical committee for animal welfare (Reg.Nr.: 15-105/08).

\section{Advanced Imaging}

Axial CT was performed to quantify RUI on virtual 3D bone models using the sphere fitting technique (Eljack et al., 2013) and to confirm absence of any joint pathology in the control group. Using the sphere fitting technique static RUI was measured at the base of the medial coronoid process. For 3D modeling, radius and ulna were manually separated from each other in the axial CT images using the open source image processing software MeVisLab (MeVis Medical Solutions AG, Bremen, Germany). This allowed generation of separate 3D surface renderings of isolated bone segments using dedicated image analysis software (VTK 3.0, Kitware Inc., NY, USA). Further enhancement, as well as inspection of the $3 \mathrm{D}$ models and quantification of RUI were performed using ParaView (Kitware, Inc., NY, USA). For CT scanning dogs were positioned in dorsal recumbence with both elbow joints extended at $\sim 135^{\circ}$. Spiral CT imaging was performed with a slice thickness of $1 \mathrm{~mm}$ and an overlapping increment of $0.5 \mathrm{~mm}$ (Philips Brilliance, Philips, Netherlands).

Only the dogs in the dysplastic group were further assessed using elbow arthroscopy, using standard medial portals and a $1.9 \mathrm{~mm}$ fore-oblique scope (Storz Endoskope, Tuttlingen, Germany). Medial compartment pathology was scored based on a modified Outerbridge scale (Fitzpatrick et al., 2009; Goldhammer et al., 2010) and fragmentation/fissuring of the medial coronoid process was assessed using palpation. Intraarticular treatment consisted solely of fragment removal. No further surgical treatment was performed, especially no resection of medial coronoid bone other than the fragment(s). Dogs were discharged the same day and oral non-steroidal anti-inflammatory drugs (NSAID) were prescribed for 1 week.

\section{Fluoroscopic Kinematography}

Along the CT scanning and/or arthroscopy, percutaneous implantation of $0.8 \mathrm{~mm}$ tantalum beads (Tantalum Beads, X-Medics Scandinavia, Frederiksberg, Denmark) into radius and ulna and humerus was performed. A minimum of 3 markers were placed into each bone. Implantation was performed with a bone marrow cannula (MarrowCut, Somatex Medical Technology, Teltow, Germany). After a small cutaneous stab incision with an 11 scalpel blade, the cannula was advanced into the cortex. The tantalum bead was inserted into the cannula and pushed into the bone tunnel created by the trocar of the cannula. Proper marker position was evaluated intraoperatively using a C-arm. After implantation of all beads, CT scanning was repeated using the same settings as for the pre-operative CT imaging.

Three weeks after bead implantation biplanar fluoroscopic kinematography of the index joint(s) was performed. By that time, none of the dogs was under NSAID or other medication. Synchronized biplanar high-speed x-ray movies of the elbow joint were taken while the dog was walking on a treadmill (Fig. 1A, B) (Jog A Dog, LLC, Michigan, USA). The bi-planar fluoroscopic setup (Fig. 2) consisted of two highpowered X-ray tubes (Philips Medio 65 CP-H X-Ray Generator, Philips, Netherlands) and two fluoroscopic image intensifiers (Philips Typ BX 3i-2123, Philips, 
Netherlands) with coupled digital high-speed video cameras (Optronis Cam Record CR600x2, Kehl, Germany). The system operated at 50-77 kV and 50-80 $\mathrm{mA}$ with an inter-beam angle of $60^{\circ}$. Video sequences of six seconds were taken at 500 frames per second, 0.5 ms shutter and a resolution of $1024 \times 1024$ pixels. The $\mathrm{x}$-ray source-to-image intensifier distance was $1.4 \mathrm{~m}$. A third synchronized high-speed video camera recorded the life video of the patient walking on the treadmill (Fig. 1C). The life video was later used to determine ground contact. At least three steps were recorded and stored using dedicated software (TimeBench, Optronis GmbH, Kehl, Germany). Every video sequence was cut to a length of 150 frames, starting 30 frames before ground contact. The remaining 120 frames represent the first $30 \%$ of stance phase.

Image distortion was corrected using a perforated steel sheet with a defined hole-diameter in XrayProject (XrayProject, Brown University, Providence, Rhode Island, USA) (Gatesy et al., 2010). Calibration of the experimental setup within the three dimensional space was also performed with XrayProject using a specified cube consisting of four layers of acrylic sheets with 64 radio-opaque markers embedded with uniform spacing (Brainerd et al., 2010).

\section{Virtual 3D Animation of elbow joint kinematics}

Tracking of 3D joint movement was performed using XrayProject. The software measures marker trajectories representing motion of the implanted markers over time by tracking the tantalum beads in both $\mathrm{x}$-ray videos. Together with the bead coordinates extracted from the CT data, 6-degree rigid-body motion ( $3 \times$ translation and $3 \times$ rotation) of radius and ulna in 3D space were calculated (Knorlein et al., 2016). Within Autodesk Maya® (Autodesk Inc., San Rafael, CA, USA), a 3D computer animation software, the calculated rigid-body motion was then applied to the previously generated CT bone models of radius and ulna, resulting in a $3 \mathrm{D}$ animation of radius and ulna which precisely mirrors the kinematics of both bones when the dog was walking on the treadmill. Finally, global radial and ulnar motions in 3D space were converted to radial movement relative to the ulna, with the ulna remaining still.

\section{Measurement of dynamic RUI}

Within Maya, a Cartesian 3D joint coordinate system was defined (Fig. 3) with the $\mathrm{z}$-axis orientated along the caudal border of the proximal ulna, the $\mathrm{x}$-axis perpendicular to the z-axis and through the tip of the anconeal process and the $y$-axis perpendicular to the first two. Dynamic RUI was defined as any radial motion along the $\mathrm{z}$-axis in relation to the ulna. Measurement of dynamic RUI started at frame 30, marking ground contact, and stopped at frame 150 .
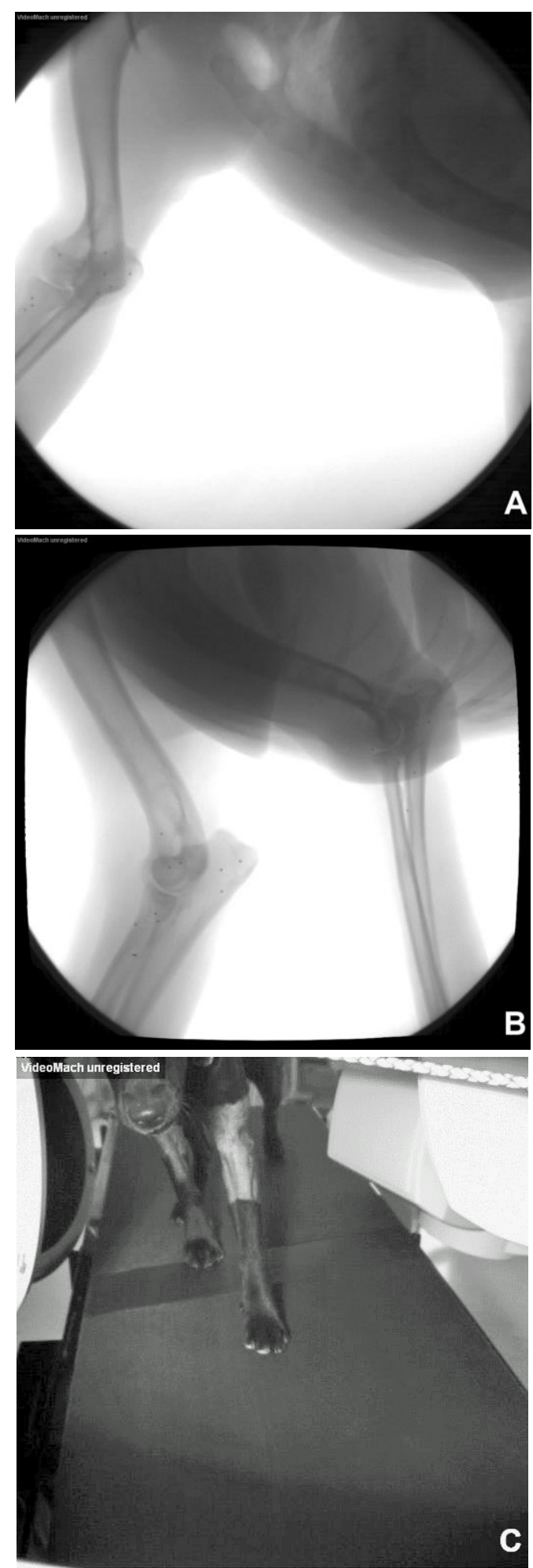

Fig. 1. Corresponding image set of synchronized bi-planar fluoroscopic high-speed video sequences $(\mathbf{A}, \mathbf{B})$ and live image camera just at the beginning of stance phase (C) for the left front limb. Implanted tantalum beads are clearly visible. All cameras operated at a frame rate of $500 / \mathrm{sec}, 0.5 \mathrm{~ms}$ shutter and a resolution of $1024 \times 1024$ pixels. 


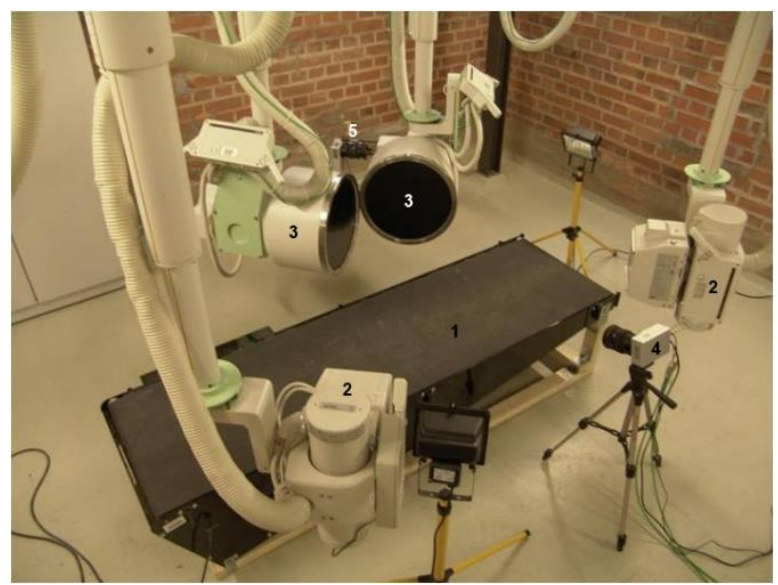

Fig. 2. Setup for high-speed bi-planar fluoroscopic kinematography. (1): treadmill; (2): x-ray tube assembly; (3): image intensifier; (4): digital high-speed video camera for live image capturing; (5): digital high-speed video cameras coupled to the image intensifier. The inter-beam angel is $\sim 60^{\circ}$ and the $\mathrm{x}$-ray source-to-image intensifier distance $1.4 \mathrm{~m}$.

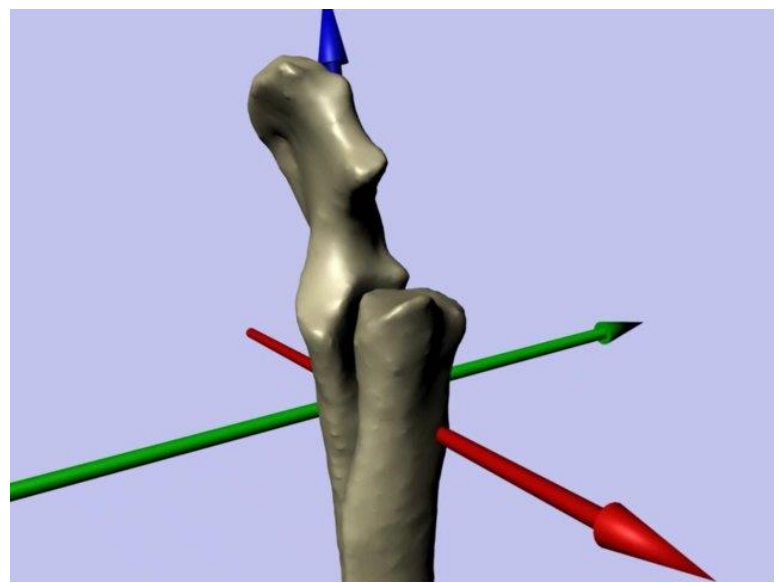

Fig. 3. Virtual bone model of the radius and ulna with embedded Cartesian joint coordinate system (cranio-medial view, left elbow joint). The z-axis (blue) is orientated along the caudal border of the proximal ulna. The x-axis (red) is orientated along the long axis of the anconeal process and the $y$-axis (green) is perpendicular to the first two axes. Dynamic radio-ulnar incongruence, expressed as axial proximo-distal translation of the radius relative to the ulna was measured along the blue axis.

The magnitude of dynamic RUI was expressed in form of the maximal amplitude of relative radio-ulnar translation (AmpRUI). For final data analysis, AmpRUI was averaged for the three step cycles of each joint (Fig. 4).

\section{Statistical analysis}

Standard descriptive statistic was performed on epidemiological data, static RUI derived from the 3D models as well as AmpRUI, and expressed as mean and standard deviation, or median and interquartile range, in case Kolmogorov-Smirnov-testing rejected the hypothesis of normality. Group wise comparison of age was done using the Mann-Whitney-U-test, while body weight, static RUI and AmpRUI were compared using an unpaired t-test. For all tests $\alpha$ was set to 0.05 . In case of significant difference, the $95 \%$ confidence interval for that difference was calculated. A software package (MedCalc, MedCalc Software, Belgium) was used to perform all analyses.

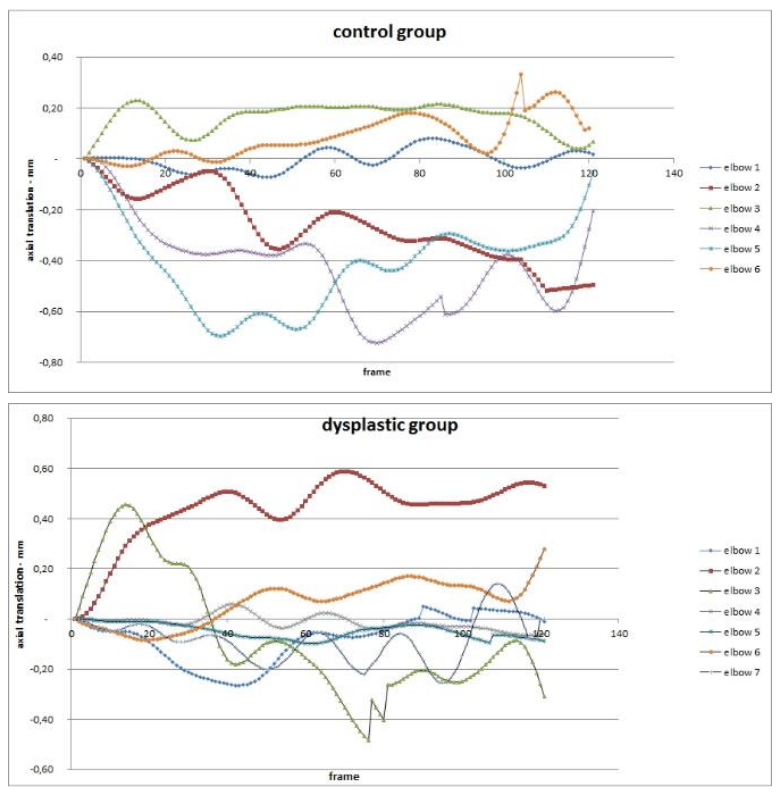

Fig. 4. Graphic depiction of axial radio-ulnar translation over 120 frames of stance phase, in healthy (control group) and dysplastic elbow joints. Frame 1 represents moment of weight bearing, defined as point zero of measurement.

\section{Animals}

\section{Results}

Five undiseased (two spayed and one intact female; two castrated males) and six dysplastic dogs (two spayed and two intact females; one castrated and one intact male) were recruited. Dogs in the control group had a median age of 17 months (IQR: $14.8-33.8$ ) and the dysplastic dogs a median age of 17.5 months (IQR: 15.0 - 21.0), not being significantly different from each other $(\mathrm{p}=0.8551)$. No significant difference was detected for body weight between groups $(\mathrm{p}=0.8551$; control: mean $27.9 \mathrm{~kg}$ [SD: 4.76]; dysplastic: $27.6 \mathrm{~kg}$ [SD: 3.98]). In the control group dog breeds included two mixed breed, and one each of Australian Shepard, Labrador retriever, and Eurasian. The dysplastic group consisted of two Labrador retriever and two mixed breed dogs, as well as one German Shepard and one Bernese mountain dog.

Only for the first dog in the control group both elbow joints where implanted with tantalum beads. This was abandoned afterwards, because time for bilateral implantation was felt to prolong anesthesia disproportionally. Overall, this led to three left and three right implanted elbow joints for the control group. 
Side of implantation was chosen by alternating left and right. For the dysplastic group, four left and three right elbow joints where recruited, as only one dog appeared to be lame on both front limbs.

\section{Clinical Data}

According to the study protocol, all control dogs were free of lameness and any pathological findings on orthopedic examination, radiographs and CT. The primary indications for general anesthesia, which was also used for tantalum bead implantation in these dogs, were castration and/or dental care.

Five of the six dysplastic dogs showed a unilateral lameness of two to eight weeks prior to presentation. The remaining dog showed lameness on both front limbs lasting already ten months. All dogs within the dysplastic group where referred to the clinic because of suspected developmental elbow disease, which was confirmed by orthopedic examination, radiography and CT of the affected elbow(s), as well as arthroscopy.

\section{Imaging Findings}

\section{Radiography and $\mathrm{CT}$}

IEWG score for the seven dysplastic joints ranged from 1 to 3 , with loss of the cranial contour of the medial coronoid process in six of seven joints. CT findings included mild to moderate osteophytosis and fragmentation/fissuring of the medial coronoid process in six of seven joints.

Mean static RUI, measured in 3D bone models based on the pre-operative CT scans, was $0.2 \mathrm{~mm}$ (SD 0.30 $\mathrm{mm}$ ) in control joints, with four/six elbow joints showing no detectable RUI. The two remaining joints had a positive RUI of $0.3 \mathrm{~mm}$ and $0.7 \mathrm{~mm}$, respectively. All seven dysplastic elbow joints had at least mild positive RUI (minimum: $0.2 \mathrm{~mm}$ ) with a mean RUI of $1.4 \mathrm{~mm}$ (SD $0.72 \mathrm{~mm}$ ). The bilaterally affected dog had an RUI of $1.9 \mathrm{~mm}$ on both sides. The highest RUI measured in the dysplastic group was $2.2 \mathrm{~mm}$. Overall, RUI in the dysplastic elbow joints was significantly greater than in the control elbows $(\mathrm{p}=0.0044,95 \% \mathrm{CI}$ : $0.5-1.9)$.

\section{Arthroscopy}

Arthroscopy confirmed medial coronoid disease in all seven joints with fragmentation/fissuring of the medial coronoid process being evident in six joints. Three joints where free of any further medial compartment pathology, except for mild synovialitis. Three other joints where affected by Outerbridge grade 2 to 3 cartilage damage on the medial coronoid process $( \pm$ humeral trochlea). Only one joint showed advanced medial compartment disease with grade 3 to 4 cartilage pathology at the medial coronoid process and humeral trochlea.

\section{Dynamic RUI}

In sound elbow joints, dynamic RUI, expressed as AmpRUI, averaged $0.7 \mathrm{~mm}$ (SD $0.31 \mathrm{~mm}$ ), while the dysplastic joints showed a mean AmpRUI of $0.5 \mathrm{~mm}$
(SD $0.30 \mathrm{~mm}$ ). Even though AmpRUI was smaller in the affected elbow joints, this difference was not significant $(p=0.2092)$.

\section{Discussion}

The current study confirms the findings of Guillou et al. (2011), who described dynamic RUI in healthy elbow joints, also using roentgen stereophotogrammetric analysis. Their reported amplitude of dynamic axial translation appears to be slightly higher $(0.9 \mathrm{~mm}$ vs. $0.7 \mathrm{~mm}$ in the current study), which could be attributed to the fact that they measured axial radio-ulnar translation along a slightly different axis and/or that they investigated dynamic RUI along the entire gait cycle, while we investigated only the first $30 \%$ of stance phase.

Irrespectively, with a standard deviation of $0.3 \mathrm{~mm}$ in the current and $0.16 \mathrm{~mm}$ in the aforementioned study with similar sample sizes, we conclude that dynamic axial radio-ulnar shift of about 0.5 to $1 \mathrm{~mm}$ can generally be expected in normal adult dogs of $20-35$ $\mathrm{kg}$, while walking or trotting. While we did not investigate trotting in the current study, Guillou et al. (2011) were unable to find significant differences between walking and trotting, which suggests that radio-ulnar motion measured at the walk could be extrapolated to trotting activity.

Our results are also in accordance with the amount of radio-ulnar translation reported in a cadaveric model of simulated triceps pull (Might et al., 2011). Therefore, the widely suspected dynamic radio-ulnar step creation during weight bearing activity (Gemmill et al., 2005; Kramer et al., 2006; Gemmill and Clements, 2007; Fitzpatrick and Yeadon, 2009, Fitzpatrick et al., 2009, 2016; Smith et al., 2009; Might et al., 2011; Gutbrod and Guerrero, 2012; Starke et al., 2013, 2014; Krotscheck et al., 2014a; Mariee et al., 2014; Eljack and Bottcher, 2015) is a consistent physiological pattern of elbow kinematic in dogs.

While the results of the current data prove the presence of dynamic RUI in canine elbow joints, the amplitude of dynamic translation was not different between normal and dysplastic joints. Therefore, excessive dynamic deformation of the radio-ulnar joint cup is unlikely to play a role in the pathogenesis of medial coronoid disease, rejecting our working hypothesis. In conclusion, dynamic RUI is truly occurring in any canine elbow joint under load, but it cannot be considered causative for the pathological changes in dysplastic elbow joint, as the amplitude of relative axial radio-ulnar translation equals the one in sound, unaffected elbow joints, at least in the first $30 \%$ of stance phase. In consequence, the discussion on dynamic RUI as the primary factor in the development of medial coronoid disease will have to be re-directed to alternative biomechanical mechanisms as an explanation for the obvious supraphysiological loading 
of the medial joint compartment in dysplastic elbow joints.

With a mean static RUI of $1.4 \mathrm{~mm}$ in the dysplastic group, we failed at recruiting two groups of dogs being comparable in every aspect considered relevant to the study. Certainly, it would have been desirable that the dysplastic dogs would have had congruent elbow joints, but on the other hand, this allows us to speculate on the significance of RUI measurement without joint load, which is inherent to the established clinical CT scanning protocols. Both normal and dysplastic elbow joints exhibit dynamic deformation of the radio-ulnar transition, but the amount of axial radio-ulnar translation did not differ in respect to the degree of static RUI. Therefore, equalization of severe static RUI during weight bearing activity appears to be unlikely. The same applies to congruent joints, which do not show dynamic RUI different to the incongruent once, and therefore can't be considered to develop a pronounced, clinically relevant incongruence throughout the weight-bearing period. In the future, more elaborated analysis of static RUI and the pattern of dynamic change will be necessary for conclusion.

\section{Limitations}

Only the first third of stance phase was evaluated in the present study, which might have resulted in loss of significant kinematic data occurring at another time point of the gait cycle, including swing phase. Limiting data analysis to the first 120 frames after foot drop was due to the fact that later during stance phase, superimposition of both elbow joints and the thoracic wall occurred at least in one fluoroscopic plane. Superimposition of both joints and the thoracic wall produced very low fluoroscopic image quality, rendering marker tracking unreliable. Nevertheless, we consider the first $30 \%$ of stance phase to be the most relevant when investigating dynamic RUI, because maximal peak vertical force in the front limbs occurs early during stance phase (Corbee et al., 2014). Therefore, we expect axial radio-ulnar translation reaching its maximum within the first third of stance phase, when the limb is maximally loaded. Another argument, further strengthening our confidence to present relevant data, is the close similarity of our kinematic findings with the one of Guillou et al. (2011), who measured axial radio-ulnar translation along the entire gait cycle, including both stance and swing phase.

Measuring only maximal amplitude (AmpRUI) of relative radio-ulnar translation, independent from the direction of radial motion, limits the prediction of the effect of radio-ulnar motion onto joint cup conformation. Proximal radial translation would result in a different radio-ulnar joint conformation than distally orientated translation. Despite no significant difference in AmpRUI values between normal and dysplastic elbow joints in the current study, individual motion patterns need to be further investigated to clarify the in vivo radio-ulnar joint cup conformation during stance phase.

Kinematic studies using treadmill walking may not mirror over ground motion, because of influence on gait pattern (Fredricson et al., 1983; Buchner et al., 1994). In humans, familiarization to the treadmill after 6 to 8 minutes has been reported. After that, gait pattern normalizes and does no longer differ from the gait on a flat underground (Schieb, 1986; Matsas et al., 2000). There are multiple studies investigating the process of familiarization to treadmill walking in dogs (Vilensky et al., 1994a, b; Owen et al., 2004; Fanchon et al., 2006) and 2 minutes turned out to be sufficient. (Owen et al., 2004) Based on this, an individual time of at least two minutes was conceded to every dog before data collection. Because dynamic RUI is assumed to be dependent on joint load, not only kinematics on the treadmill should mimic the once during over ground activity, but also the kinetics. The latter has been confirmed by Brebner et al. (2006) who documented a good correlation of ground reaction forces on an instrumented treadmill and the one with a force plate embedded in the floor.

In conclusion, the results of this study show that the concept of in vivo dynamic RUI applies to both normal and dysplastic canine elbow joints. However, dynamic RUI is not different in dysplastic joints compared to normal, and therefore cannot be considered relevant for the development of medial compartment disease. In addition, compensation of severe static radio-ulnar incongruence in dysplastic elbow joints as well as the dynamic induction of radio-ulnar incongruence in statically congruent joints seems unlikely. However, this latter statement will need verification using more elaborated kinematic analysis as presented in the actual study.

\section{Acknowledgments}

The authors would like to thank the medical as well as the technical stuff of both, the imaging and aesthesia service, for their generous support throughout the study.

\section{Conflict of interest}

The authors declare that there is no conflict of interests.

\section{References}

Brainerd, E.L., Baier, D.B., Gatesy, S.M., Hedrick, T.L., Metzger, K.A., Gilbert, S.L. and Crisco, J.J. 2010. X-ray reconstruction of moving morphology (XROMM): precision, accuracy and applications in comparative biomechanics research. J. Exp. Zool. A. Ecol. Genet. Physiol. 313, 262-279.

Brebner, N.S., Moens, N. and Runciman, J. 2006. Evaluation of a treadmill with integrated force plates for kinetic gait analysis of sound and lame 
dogs at a trot. Vet. Comp. Orthop. Traumatol. 19, 205-212.

Buchner, H.H., Savelberg, H.H., Schamhardt, H.C., Merkens, H.W. and Barneveld, A. 1994. Kinematics of treadmill versus overground locomotion in horses. Vet. Q. 16(Suppl 2), S87-90.

Corbee, R.J., Maas, H., Doornenbal, A. and Hazewinkel, H.A.W. 2014. Forelimb and hindlimb ground reaction forces of walking cats: Assessment and comparison with walking dogs. Vet. J. 202, 116-127.

Eljack, H. and Bottcher, P. 2015. Relationship between axial radioulnar incongruence with cartilage damage in dogs with medial coronoid disease. Vet. Surg. 44, 174-179.

Eljack, H., Werner, H. and Bottcher, P. 2013. Sensitivity and specificity of 3D models of the radioulnar joint cup in combination with a sphere fitted to the ulnar trochlear notch for estimation of radioulnar incongruence in vitro. Vet. Surg. 42, 365-370.

Fanchon, L., Valette, J.P., Sanaa, M. and Grandjean, D. 2006. The measurement of ground reaction force in dogs trotting on a treadmill: an investigation of habituation. Vet. Comp. Orthop. Traumatol. 19, 8186.

Fitzpatrick, N. and Yeadon, R. 2009. Working algorithm for treatment decision making for developmental disease of the medial compartment of the elbow in dogs. Vet. Surg. 38, 285-300.

Fitzpatrick, N., Smith, T.J., Evans, R.B. and Yeadon, R. 2009. Radiographic and arthroscopic findings in the elbow joints of 263 dogs with medial coronoid disease. Vet. Surg. 38, 213-223.

Fitzpatrick, N., Garcia, T.C., Daryani, A., Bertran, J., Watari, S. and Hayashi, K. 2016. Micro-CT Structural Analysis of the Canine Medial Coronoid Disease. Vet. Surg. 45, 336-346.

Fredricson, I., Drevemo, S., Dalin, G., Hjerten, G., Bjorne, K., Rynde, R. and Franzen, G. 1983. Treadmill for equine locomotion analysis. Equine Vet. J. 15, 111-115.

Gatesy, S.M., Baier, D.B., Jenkins, F.A. and Dial, K.P. 2010. Scientific rotoscoping: a morphology-based method of 3-D motion analysis and visualization. J. Exp. Zool. A Ecol. Genet. Physiol. 313, 244-261.

Gemmill, T.J. and Clements, D.N. 2007. Fragmented coronoid process in the dog: is there a role for incongruency? J. Small Anim. Pract. 48(7), 361368.

Gemmill, T.J., Mellor, D.J., Clements, D.N., Clarke, S.P., Farrell, M., Bennett, D. and Carmichael, S. 2005. Evaluation of elbow incongruency using reconstructed CT in dogs suffering fragmented coronoid process. J. Small Anim. Pract. 46, 327333.
Goldhammer, M.A., Smith, S.H., Fitzpatrick, N. and Clements, D.N. 2010. A comparison of radiographic, arthroscopic and histological measures of articular pathology in the canine elbow joint. Vet. J. 186, 96-103.

Guillou, R.P., Déjardin, L.M., Bey, M.J. and McDonald, C.P. 2011. Three Dimensional Kinematics of the Normal Canine Elbow at the Walk and Trot. 2011 American College of Veterinary Surgeons Veterinary Symposium November 3-5, Chicago, Illinois. Vet. Surg. 40, E17-E42.

Gutbrod, A. and Guerrero, T.G. 2012. Effect of external rotational humeral osteotomy on the contact mechanics of the canine elbow joint. Vet. Surg. 41, 845-852.

Kirberger, R.M. and Fourie, S.L. 1998. Elbow dysplasia in the dog: pathophysiology, diagnosis and control. J. South African Vet. Assoc. 69, 43-54.

Knorlein, B.J., Baier, D.B., Gatesy, S.M., LaurenceChasen, J.D. and Brainerd, E.L. 2016. Validation of XMALab software for marker-based XROMM. J. Exp. Biol. 219, 3701-3711.

Kramer, A., Holsworth, I.G., Wisner, E.R., Kass, P.H. and Schulz, K.S. 2006. Computed tomographic evaluation of canine radioulnar incongruence in vivo. Vet. Surg. 35, 24-29.

Krotscheck, U., Bottcher, P.B., Thompson, M.S., Todhunter, R.J. and Mohammed, H.O. 2014a. Cubital subchondral joint space width and CT osteoabsorptiometry in dogs with and without fragmented medial coronoid process. Vet. Surg. 43, 330-338.

Krotscheck, U., Kalafut, S., Meloni, G., Thompson, M.S., Todhunter, R.J., Mohammed, H.O. and van der Meulen, M.C.H. 2014b. Effect of Ulnar Ostectomy on Intra-Articular Pressure Mapping and Contact Mechanics of the Congruent and Incongruent Canine Elbow Ex Vivo. Vet. Surg. 43, 339-346.

Lau, S.F., Hazewinkel, H.A. and Voorhout, G. 2015. Radiographic and computed tomographic assessment of the development of the antebrachia and elbow joints in Labrador Retrievers with and without medial coronoid disease. Vet. Comp. Orthop. Traumatol. 28, 186-192.

Mariee, I.C., Gröne, A. and Theyse, L.F.H. 2014. The role of osteonecrosis in canine coronoid dysplasia: Arthroscopic and histopathological findings. Vet. J. 200, 382-386.

Mason, D.R., Schulz, K.S., Fujita, Y., Kass, P.H. and Stover, S.M. 2005. In vitro force mapping of normal canine humeroradial and humeroulnar joints. Am. J. Vet. Res. 66, 132-135.

Matsas, A., Taylor, N. and McBurney, H. 2000. Knee joint kinematics from familiarised treadmill 
walking can be generalised to overground walking in young unimpaired subjects. Gait Posture 11, 4653.

McConkey, M.J., Valenzano, D.M., Wei, A., Li, T., Thompson, M.S., Mohammed, H.O., van der Meulen, M.C.H. and Krotscheck, U. 2016. Effect of the Proximal Abducting Ulnar Osteotomy on IntraArticular Pressure Distribution and Contact Mechanics of Congruent and Incongruent Canine Elbows Ex Vivo. Vet. Surg. 45, 347-355.

Might, K.R., Hanzlik, K.A., Case, J.B., Duncan, C.G., Egger, E.L., Rooney, M.B. and Duerr, F.M. 2011. In Vitro Comparison of Proximal Ulnar Osteotomy and Distal Ulnar Osteotomy with Release of the Interosseous Ligament in a Canine Model. Vet. Surg. 40, 321-326.

Morgan, J.P., Wind, A. and Davidson, A.P. 1999. Bone dysplasias in the labrador retriever: a radiographic study. J. Am. Anim. Hosp. Assoc. 35, 332-340.

Nemanic, S., Nixon, B.K. and Baltzer, W. 2016. Analysis of risk factors for elbow dysplasia in giant breed dogs. Vet. Comp. Orthop. Traumatol. 29, 369-377.

Ohlerth, S., Tellhelm, B., Amort, K. and Ondreka, N. 2016. Explanation of the IEWG grading system, In: $30^{\text {th }}$ annual meeting of the INTERNATIONAL ELBOW WORKING GROUP, Vienna, Austria.

Owen, M., Richards, J., Clements, D., Drew, S., Bennett, D. and Carmichael, S. 2004. Kinematics of the elbow and stifle joints in greyhounds during treadmill trotting-An investigation of familiarisation. Vet. Comp. Orthop. Traumatol. 17, 141.

Samoy, Y., Van Ryssen, B., Gielen, I., Walschot, N. and van Bree, H. 2006. Review of the literature: elbow incongruity in the dog. Vet. Comp. Orthop. Traumatol. 19, 1-8.

Schieb, D.A. 1986. Kinematic accommodation of novice treadmill runners. Res. Q. Exerc. Sport 57, 1-7.

Smith, T.J., Fitzpatrick, N., Evans, R.B. and Pead, M.J. 2009. Measurement of Ulnar Subtrochlear Sclerosis Using a Percentage Scale in Labrador Retrievers with Minimal Radiographic Signs of Periarticular Osteophytosis. Vet. Surg. 38, 199-208.

Starke, A., Bottcher, P. and Pfeil, I. 2013. Radiologic quantification of the elbow conformation with a new method for acquiring standardized $\mathrm{x}$-rays under load. Reference valus for medium sized and large dogs without dysplasia of the elbow joint. Tierarztliche Praxis. Ausgabe K, Kleintiere/Heimtiere 41, 145-154.

Starke, A., Bottcher, P. and Pfeil, I. 2014. Comparative radiologic examination of the canine elbow with and without elbow dysplasia under standardized load. Tierarztliche Praxis. Ausgabe K, Kleintiere/Heimtiere 42, 141-150.

Vilensky, J.A., O'Connor, B.L., Brandt, K.D., Dunn, E.A. and Rogers, P.I. 1994a. Serial kinematic analysis of the trunk and limb joints after anterior cruciate ligament transection: Temporal, spatial, and angular changes in a canine model of osteoarthritis. J. Electromyogr. Kinesiol. 4, 181192.

Vilensky, J.A., O'Connor, B.L., Brandt, K.D., Dunn, E.A., Rogers, P.I. and Delong, C.A. 1994b. Serial kinematic analysis of the unstable knee after transection of the anterior cruciate ligament: temporal and angular changes in a canine model of osteoarthritis. J. Orthop. Res. 12, 229-237. 